\title{
TTR
}

Traduction, terminologie, re?daction

\section{La poésie pense : une modalité assomptive de la connaissance}

\section{Annie Brisset}

Volume 12, numéro 1, 1er semestre 1999

Poésie, cognition, traduction I

Poetry, Cognition, Translation I

URI : https://id.erudit.org/iderudit/037350ar

DOI : https://doi.org/10.7202/037350ar

Aller au sommaire du numéro

\section{Éditeur(s)}

Association canadienne de traductologie

ISSN

0835-8443 (imprimé)

1708-2188 (numérique)

Découvrir la revue

Citer cet article

Brisset, A. (1999). La poésie pense : une modalité assomptive de la connaissance. TTR, 12(1), 9-18. https://doi.org/10.7202/037350ar

\section{Résumé de l'article}

La poésie pense : une modalité assomptive de la connaissance - Dans un monde utilitariste dominé par la technologie, la poésie ne correspond pas forcément aux idées qu'on a sur elle. Exploration du monde et de la vie, elle est comme la science au coeur de la connaissance, repoussant elle aussi les limites de l'inconnu et de l'impensé. Elle est encore la conscience critique du rapport nécessairement subjectif à la connaissance. C'est par son caractère " épiphanique » que la poésie se distingue du discours scientifique. Au savoir-vrai argumenté du discours de la science, elle oppose la modalité assomptive, immédiate, de la saisie du monde.

Tous droits réservés ( $\mathrm{C}$ TTR: traduction, terminologie, rédaction — Les auteurs, Ce document est protégé par la loi sur le droit d’auteur. L’utilisation des 1999 d'utilisation que vous pouvez consulter en ligne.

https://apropos.erudit.org/fr/usagers/politique-dutilisation/ 


\title{
La poésie pense1 : une modalité assomptive de la connaissance
}

\author{
Annie Brisset
}

Il faut représenter l'homme dans sa totalité, dans toute la gamme de ses expériences vécues, en allant de ses possibilités physiques et de ses sentiments au domaine moral et métaphysique, d'où un appel immédiat au lyrisme, seul capable d'en fournir l'expression.

Hermann Broch (tr. A. Picard, 1961, p. 290)

Dans un monde dominé par la technologie, oủ l'on tend à privilégier la fonction utilitaire de la traduction et à sous-estimer les textes qui ne s'identifient pas immédiatement avec la technoscience ou les discours de savoir, il est important de montrer que la production poétique ne correspond pas forcément aux idées qu'on a sur elle :

Le sociologue et le psychologue ont beau serrer de plus en plus leurs réseaux de connaissances, toucher de plus en plus près a l'objectivité, ils seront toujours des observateurs, c'est-à-dire situés en pleine subjectivité. Tous les savants en sont là. Quant aux philosophes, ils ne semblent pas près d'établir une équation satisfaisante entre ces deux faces de Janus. Un seul homme peut prétendre avoir quelque connaissance de la vie dans ce qui fait sa substance, le poète; parce qu'il se tient au cour du drame qui se joue entre ces deux pôles: objectivité-subjectivité; parce qu'il les exprime à sa manière qui est le déchirement, dont il se nourrit luimême et dont, quant au monde, il est le porte-venin ou si l'on veut, porte-parole. (Leiris, 1934, p. 210)

La poésie, exploration du monde et de la vie est, comme la science, au cour de la connaissance, repoussant elle aussi les limites de l'inconnu et de l'impensé. La question n'est pas de reprendre, banalement, le débat qui les oppose depuis très longtemps, avec « le

\footnotetext{
${ }^{1}$ L'expression est de Henri Meschonnic.
} 
duo de la science-objectivité, et de la poésie-subjectivité, la subjectivité au sens d'un arbitraire narcissique, qui prend le moi pour le je, et se préfère à tout" (Meschonnic, 1994, p. 30). La question est de voir comment la poésie pense. Le poète est-il le " porte-venin " du monde, la conscience critique du rapport nécessairement subjectif à la connaissance?

La connaissance est l'aboutissement provisoire d'un processus, celui de l'appropriation d'un objet, d'un fait, d'un phénomène, d'une manière de comprendre. Elle est médiatisée par un savoir antérieur qui s'incarne dans différents discours. Par eux transite l'intentionalité de l'énonciateur, sa tentative de produire, de manipuler, d'organiser, de recevoir et de manifester un savoir. Mais la valeur cognitive du discours médiateur, du savoir médiateur, est compromise par le cadrage des préconstruits que ce discours impose. Tous les discours possèdent une dimension cognitive, régie selon Barthes par au moins trois forces : mathésis, mimésis, sémiosis, c'est-à-dire le savoir, la représentation et la production de sens $(1977$, p. 17). La poésie a ceci de particulier qu'elle exerce ces trois forces sur les matériaux discursifs eux-mêmes :

Parce qu'elle met en scène le langage, au lieu, simplement, de l'utiliser, [la littérature] engrène le savoir dans le rouage de la réflexivité infinie : à travers l'écriture, le savoir réfléchit sans cesse sur le savoir, selon un discours qui n'est plus épistémologique, mais dramatique. [...] Il est de bon ton, aujourd'hui, de contester l'opposition des sciences et des lettres [...]; et il est possible que cette opposition apparaisse un jour comme un mythe historique. Mais du point de vue du langage, qui est le nôtre ici, cette opposition est pertinente; ce qu'elle met en regard n'est d'ailleurs pas forcément le réel et la fantaisie, l'objectivité et la subjectivité, le Vrai et le Beau, mais seulement des lieux différents de parole. Selon le discours de la science -, le savoir est un énoncé; dans l'écriture il est une énonciation. [...] L'énonciation, elle, en exposant la place et l'énergie du sujet, voire son manque (qui n'est pas une absence), vise le réel même du langage. (Barthes, 1977, pp. 19-20)

La poésie est consubstantielle à cette mise en perspective cognitive. Il y entre, par voie conventionnellement surcodée, un savoir sur la poésie, mais un savoir reconduit chaque fois qu'advient un poème, une subjectivité, celle d'un moi " lyrique ». Il y entre le sujet humain dans ses rapports au monde, à l'autre et à la langue - au cosmos, à l'anthropos et au logos, pour reprendre le modèle triadique du groupe Mu (1977). C'est aussi l'idée que Meschonnic exprime en ces termes: "Les grands inventeurs sont des artistes du savoir, comme les poètes - les vrais - sont des savants du quotidien, des apprentis de l'imperceptible qui transforment les rapports que nous avons avec le langage, avec nous-mêmes, avec la poésie déjà écrite et avec la poésie à écrire. C'est cette transformation que j'appelle la poésie » (p. 30). 
Dans son esquisse d'une typologie des discours cognitifs, A. J. Greimas (1979, p. 42) distingue les « discours interprétatifs ", comme la critique littéraire, la critique d'art ou l'histoire, les "discours persuasifs", comme ceux de la pédagogie, de la politique ou de la publicité, et enfin les "discours scientifiques" axés sur le savoir-vrai et qui jouent à la fois sur la persuasion (avec le jeu de la démonstration) et sur l'interprétation (en exploitant les discours antérieurs comme discours de référence). Les discours artistiques ne figurent pas dans cette typologie, comme si ce que Lotman appelle leur "artisticité " - modélisation secondaire du réel - n'entrait pas, en tant que telle, dans le registre de la cognition. Mais rien ne nous empêche de les y introduire. Avançons que l'épiphanie est le mode cognitif qui leur est propre et appelons-les discours assomptifs. L'extrait suivant d'un poème de Hölderlin et son commentaire par William H. Gass (1999) servira d'illustration :

Mit gelben Birnen hänget

Und voll mit wilden Rosen

Das Land in den See.

"With yellow pears hangs / And full with wild roses / the land in the lake. " Easily said, less easily understood, because the order of the words is - well - wild as the roses are. (p. 47)

Ces trois vers, observe W. H. Gass, renvoient le lecteur à l'expérience. On voit les branches ployer sous le poids des fruits, le reflet inversé des buissons et des arbres traversant la surface de l'eau. Et retournant au titre du poème (Hälfte des Lebens / Moitiés de la vie), on comprend que les fruits et leur image sont la moitié réelle d'un tout imaginé : l'objet et son reflet conjoints. Pourquoi, demande W. H. Gass, l'ordre naturel de la phrase est-il modifié? La modification, en vérité, ne porte que sur un élément. Le verbe - hänget - est déplacé, mais il est déplacé de telle sorte qu'il anticipe un sujet et un objet, puis fait résonner son écho en chacun d'eux. Cet élément de perturbation et d'unification rythme le sens et sa lecture : "La "flèche du temps" a un rôle créateur de structures. Nous ne pouvons parler de "système" que dans les situations de non-équilibre" (Prigogine, 1994, p. 35). La moitié de la vie est vécue. Chargée de fruits délicieux, elle contemple son avenir, mais un avenir où le présent, alors passé, n'est plus qu'un objet de mémoire. Le reflet dans l'eau est fidèle, mais ce n'est qu'une image. Adoptant la position du poète, nous nous voyons tels que nous sommes à présent et tels que nous serons : une illusion. W. H. Gass résume sa "translecture " de la strophe: "What a beautiful idea : earth, solid and settled, flesh rosy and trim, life full and accomplished, altering into water, into remembrance, into image » (p. 48). Cette épiphanie, cette vision est induite par un déséquilibre, à la manière dont la poiesis émerge des figures du chaos dans le monde physique, comme le montre encore Prigogine (p. 29): "J'aime à dire que la matière à 
l'équilibre est aveugle, chaque molécule ne "percevant" que les premières molécules qui l'entourent. Par contraste, le non-équilibre conduit la matière à "voir". Une nouvelle cohérence émerge alors 》.

Si la poésie permet la connaissance, c'est que son statut ontologique n'a pas grand-chose à voir avec un mythe entretenu par la critique et qui a fini par s'enraciner dans le monde de la traduction dite pragmatique. C'est une idéologie qui voit surtout dans la poésie un art de l'ornementation. Il est vrai qu'avant de lui reconnaître une place dans l'échelle des valeurs cognitives, beaucoup de théoriciens, célèbres et moins célèbres, n'y ont vu qu'artifices verbaux, jeux de parallélismes ou figures de style. Cet état de choses a inévitablement marqué les discours et les pratiques de la traduction poétique ${ }^{2}$. Dans les approches dérivées de la linguistique, la tendance est de réduire les contenus cognitifs à des contenus référentiels dont on met en avant la permanence et l'universalité, non sans les banaliser dans le même souffle, tandis que la forme porterait à elle seule la "charge " esthétique. Malgré les tentatives d'u intégration » (Snell-Hornby) et ici l'apport de la linguistique cognitive, la dichotomie subsiste, tributaire encore de ce que Derrida appelle « cette vieille linguistique du mot » :

[...] equivalence in literary translation should be considered, and ultimately defined, in terms of poetics. [...] I will take " poetics " to mean what Jakobson took it to mean : a particular function of language; the way of structuring information within a text, and an integral part of linguistics. [...] Poetics of translation will then deal with equivalence considered on this particular level : the level of textual structure. [...] Equivalence of " poetic value " of a text [...] will be concerned with the relation between "sameness" and " difference " [...] seen - as the proponents of cognitive linguistics want to see it - as " that dialectic tension which is a central problem of translation " (Snell-Hornby 1988, p. 19). It is precisely at the point where " sameness " and " difference " meet that poetics imperceptibly melts into "stylistics" so that for all practical purposes, the two become undistinguishable. [...] Within the CL [cognitive linguistics] framework, "sameness " pertains to the universal nature of human cognition, while "difference " is the property of an infinite variety of products of cognitive processes. (Tabakowska, 1993, pp. 3-4).

Encadré par le "répertoire des modes d'expression" où s'exercent les « choix possibles » (p. 4), on peut douter que le "style » suffise à couvrir les "formes-sens" (Meschonnic) de la textualité poétique dans toute la diversité de ses manifestations.

\footnotetext{
${ }^{2}$ Pour une présentation des différents courants, voir I. Oseki-Dépré, 1999. On y souligne en particulier la dualité qui oppose une " tendance poétique ", attentive $\mathrm{d}$ " la facture (le poïen) 》 et une « tendance esthétique » qui recherche " le plaisir esthétique (l'aesthesis) »(p. 76).
} 
Au regard d'un autre discours qui affirme la "beauté " ahistorique et indifférenciée de la poésie, on ne saurait trop rappeler ce fait d'évidence : " il n'y a pas "la" poésie, mais des poèmes. Comme il n'y a pas "la" science, mais "les" sciences" (Meschonnic, p. 31). Pluralité externe accentuée par la pluralité interne, car chaque poème est différent des autres :

Dans le champ de la modernité $[. .$.$] c'est par une torsion du$ discursif sur le subjectif que la poésie se transforme, décloisonne les catégories critiques comme la strophe, le vers, l'image, la métaphore et jusqu'à celle de poème. On voit se constituer le langage poématique plutôt que le langage poétique, car la poésie moderne se déplace précisément de la poésie vers le poème. Plus l'intensité du subjectif intervient dans le poème, plus celui-ci joue sa poéticité indépendamment des structures fixes ou en tout cas par une certaine distance vis-à-vis d'elles, (Krysinski, 1995, 183)

Chaque poème manifeste son indépendance par la structure cognitive qui lui est propre. Chaque poème a ses "points de bifurcation ", suscitant la probabilité de nouvelles assomptions de connaissance. Variable en forme et en substance, la poésie a subi de nombreuses transformations. Ainsi, dans notre modernité, qui accuse déjà quelques siècles, on a vu le poétique se déplacer vers le narratif, par exemple chez Joyce, Virginia Woolf, Hermann Broch ou Claude Simon. Et on a vu le mouvement inverse : chez Apollinaire, Cendrars ou T.S. Eliot, c'est le narratif qui se déplace vers le poétique. Par ailleurs, le lyrisme de Pétrarque ne se confond pas avec celui de Baudelaire ou avec celui de Rilke. À l'origine de la poésie dite lyrique, Dilthey pose «l'unité de l'intérieur et de l'extérieur, de la vie et de la forme ", unité qui devient le " véhicule de la vision du monde et même de la pensée philosophique » (1947 [1887], p. 120). À cette loi qu'il énonce, Dilthey donne le nom de " loi schillérienne ». Elle consiste en ceci que le « processus esthétique saisit dans la forme la vie connue par le sentiment et anime ainsi les représentations ou exprime cette vie par des représentations et transporte ainsi la vie dans la forme de telle sorte que l'événement intime se traduit constamment en forme et la forme en événement intime » (p. 168, et p. 180). La traduction des expériences de la vie en images définit non seulement la dimension esthétique mais aussi la dimension cognitive du poème. Si le principe de savoir qui soutient le poème réside dans le système de ses images (Bildzusammenhang), leur structure cognitive varie de façon idiosyncratique, car elles supposent un rapport individué au réel. Prenons l'exemple de Baudelaire. Walter Benjamin relève que l'« effondrement de l'aura » qui caractérise cette cuvre serait liée à l'image dialectique qui en régit le style poétique et la modalité cognitive. Benjamin définit l'aura d'un objet comme "l'ensemble des images qui, surgies de la mémoire involontaire, tendent à se regrouper autour de lui ». Cette mémoire est le résultat de l'expérience. Celle de la foule par exemple : 
Tout à l'heure, comme je traversais le boulevard, en grande hâte, et que sautillais dans la boue, à travers ce choc mouvant où la mort arrive au galop de tous les côtés à la fois, mon auréole, dans un mouvement brusque, a glissé de ma tête dans la fange du macadam. Je n'ai pas eu le courage de la ramasser. J'ai jugé moins désagréable de perdre mes insignes que de me faire rompre les os. Et puis maintenant, me suis-je dit, à quelque chose malheur est bon. Je puis maintenant me promener incognito, faire des actions basses, et me livrer à la crapule comme les simples mortels. Et me voici, tout semblable à vous, comme vous voyez $!^{3}$

L'expérience physique de la foule, c'est, note Benjamin, « le prix que l'homme moderne doit payer pour sa sensation: l'effondrement de l'aura dans l'expérience vécue du choc » :

Perdu, dans ce vilain monde, coudoyé par les foules, je suis comme un homme lassé dont l'ceil ne voit en arrière, dans les années profondes, que désabusement et amertume, et, devant lui, qu'un orage où rien de neuf n'est contenu, ni enseignement, ni douleur ( $p$. 206).

Benjamin ajoute que « la connivence de Baudelaire avec cet effondrement lui a coûté cher. Mais c'est la loi de sa poésie " (p. 207). On en prend la mesure dans un poème comme La fontaine de sang où la fusion d'éléments contradictoires dans une image qui marque la pression du réel sur l'affectif aboutit à une connaissance immédiate, fulgurante, de la réalité :

Il me semble parfois que mon sang coule à flots,

Ainsi qu'une fontaine aux rythmiques sanglots.

Je l'entends bien qui coule avec un long murmure,

Mais je me tâte en vain pour trouver la blessure.

À travers la cité, comme dans un champ clos,

Il s'en va, transformant les pavés en îlots,

Désaltérant la soif de chaque créature,

Et partout colorant en rouge la nature.

J'ai demandé souvent à des vins capiteux

D'endormir pour un jour la terreur qui me mine;

Le vin rend l'œil plus clair et l'oreille plus fine !

J'ai cherché dans l'amour au sommeil oublieux;

Mais l'amour n'est pour moi qu'un matelas d'aiguilles

Fait pour donner à boire à ces cruelles filles !

(Baudelaire, 1991 [1857], p. 163).

${ }^{3}$ Le Spleen de Paris, « Perte d'auréole ». Cité par Benjamin, 1979 [1955], p. 205. 
La vie moderne, l'expérience de l'espace urbain, est source de blessures, et l'amour même source d'échec. L'excès et l'absence de cause sont le ressort dialectique et le principe constructeur des images : " mon sang coule à flots »/《 mais je tâte en vain ma blessure ", " une fontaine »/《 aux rythmiques sanglots 》, " l'amour »// un matelas d'aiguilles ». Dans cette intériorisation d'une expérience extérieure, la " loi schillérienne » est encore à l'œuvre.

Mise en scène discursive de l'expérience de la vie, la poésie est inséparable de la connaissance. A l'issue de cette transformation, les images font du poème un signe et son message. Elles virtualisent, comme ici encore chez Rilke (1962 [1905], p. 104), la puissance assomptive du poème :

HERR : Wir sind ärmer denn die armen Tiere, die ihres Todes enden, wennauch blind, weil wir noch alle ungestorben sind.

Den gieb uns, der die Wissenschaft gewinnt, das Leben aufzubinden in Spaliere, um welche zeitiger der Mai beginnt.

\begin{abstract}
Seigneur : nous sommes plus pauvres encor que les plus pauvres bêtes qui, bien qu'aveugles, meurent leur mort, parce qu'aucun de nous n'est encore jamais mort. Oh! donne-nous celui qui trouvera la science de lier la vie en espaliers pour lesquels fleurira un printemps plus précoce. (tr. J. Legrand, Rilke, 1972 [1929], p. 115).
\end{abstract}

Le discours assomptif, c'est aussi la présence d'une réflexion : "Le niveau réflexif très avancé sur lequel se meut la poésie de Rilke ne contrecarre en rien la maîtrise de l'invention poétique. Le sens de l'énoncé et le mode du dire se recouvrent en tous points, et comme ce sens s'avère être d'une pénétration philosophique remarquable, pensée et poésie semblent ici être indissociablement unies » (de Man, 1972, p. 12). On note que cette description du ressort cognitif de la poésie rejoint la notion de "forme-sens » évoquée plus haut, qui sous-tend l'épistémologie de l'écriture et la poétique de la traduction chez Henri Meschonnic (1973, pp. 180-181).

Les identités que l'on attribue ou que l'on refuse à une écriture ou à un texte infléchissent les modalités de sa traduction. On a dit que la traductologie prête souvent à la poésie un ensemble d'attributs qui tendent à réduire et même à nier sa dimension cognitive. Le problème se situe, comme on a pu s'en apercevoir, dans la conception même du traduire telle que nous l'expose Jakobson - et avant lui Heidegger : 
La phénoménologie de la traduction est le principal adversaire théorique. Son essentialisation du sens, chez Heidegger, n'est pas séparable de la phénoménologie du comprendre. Heidegger l'a explicité dans plusieurs passages. Dans Parménide [...] " comprendre est déjà traduire ", il y a un " traduire originel » dans le rapport même qui fait le sens. [...] Traduire est donc dilué analogiquement dans l'histoire de l'interprétation. Ce qui a pour effet de poser cette banalité spécieuse ou tout au moins insuffisante que pour comprendre il faut interpréter et pour traduire il faut d'abord avoir compris. Donc la traduction est nécessairement une interprétation. Il y a à secouer cette vérité trop bien établie, non pas pour espérer la faire tomber, mais au moins lui faire rendre ce qu'elle contient et ne montre pas. (Meschonnic, 1999, pp. 74-75)

Dans son texte séminal sur les aspects linguistiques de la traduction, Jakobson développe le même argumentaire : la traduisibilité est au principe de la saisie du sens; comprendre, c'est déjà traduire. Traduire, c'est dire autrement en vertu de l'arbitraire du signe qui permet l'«équivalence » de signifiants interchangeables - ou presque - escamotant de toute façon la signifiance, c'est-à-dire le dispositif ou le projet sémiotique du texte. Les tenants d'une « théorie interprétative » aux multiples variantes ont emboîté le pas, aveugles à ce qui sépare le texte et la langue, la "forme-sens » et le signifié, le cognitif et le dénotatif. La traductologie est passée sans grande transition du modèle interprétatif, ancré (à son insu) dans une linguistique du signe, à l'étude des faits accomplis (et souvent peu glorieux) de la traduction. Jamais on aura autant parlé de l'éthique du traduire. Entre les deux, la linguistique textuelle appliquée par la théorie du Skopos effleure gentiment la texture du sens (prenant par exemple, comme le fait Christiane Nord, l'isotopie pour un simple réseau lexico-thématique). Au moment où, sortant des problèmes linguistiques, la traductologie allait rejoindre la sémiotique, l'organicité cognitive des mises en discours, elle s'est engouffrée dans le créneau des "manipulations" idéologiques, en même temps que l'informatisation des procédures de traduction la faisait bifurquer vers la terminologie, la ramenant de nouveau vers le signe. À quelques exceptions près - parmi lesquelles Meschonnic occupe une place importante (en dépit du caractère sentencieux, idéologisé, de son écriture), parmi lesquelles on peut encore situer Berman (1995) -, le territoire de la textualité comme poüesis demeure non pas inconnu mais largement inexploré dans le champ propre de la traductologie.

À la faveur d'une réflexion philosophique qui se déploie depuis plusieurs années autour de la nature autopoiétique du virtuel (voir par exemple les travaux de Pierre Lévy), la fonction cognitive du texte poétique nous est rappelée de façon imprévisible. Ce nouveau regard sur notre "écologie cognitive" incite à revoir certains présupposés qui touchent aussi bien les genres que les modalités de traduction. Pour contribuer à cette remise en cause, les textes rasssemblés dans ce numéro interrogent les modalités du savoir dans la 
poésie, la question du sujet, la fonction cognitive de l'image, celle de l'intertexte et des structures ludiques. Autant d'aspects qu'il sera utile de confronter aux pratiques du traduire et aux positions diverses de la critique des traductions.

\section{Université d'Ottawa}

\section{Références}

BARTHES, Roland (1977). Leçon. Paris, Seuil.

BAUDELAIRE, Charles (1991 [1857]). Les Fleurs du mal. Paris, Flammarion.

BENJAMIN, Walter (1979 [1955]). Charles Baudelaire. Un poète lyrique à l'apogée du capitalisme. Paris, Payot.

BERMAN, Antoine (1995). Pour une critique des traductions : John Donne. Paris, Gallimard.

BROCH, Hermann (1961). « Genèse du livre ». Les Irresponsables. Tr. A. Picard, Paris, Gallimard.

DE MAN, Paul, éd. (1972). "Introduction ». Rainer Maria Rilke. CEuvres 2. Poésie. Paris, Seuil.

DILTHEY, Wilhelm (1947 [1887]). "L'imagination poétique 》. Le Monde de l'esprit. Tr. M. Remy, Paris, Aubier-Montaigne.

GASS, William H. (1999). Reading Rilke. Reflections on the Problems of Translation. New York, Alfred A. Knopf.

GREIMAS, Algirdas J. (1979). "Cognition 》. Sémiotique. Dictionnaire raisonné de la théorie du langage. Paris, Hachette.

GROUPE Mu (1977). Rhétorique de la poésie. Bruxelles, Éditions Complexe.

KRYSINSKI, Wladimir (1995). "Grammaire de la poésie et subjectivité dans le poème ». P. Laurette et H. G. Ruprecht (éds.), Poétiques et imaginaires. Francopolyphonie littéraire des Amériques. Paris, L'Harmattan, pp. 181-189.

LEIRIS, Michel (1934). L'Afrique fantôme. Paris, Gallimard. 
MESCHONNIC, Henri (1999). Poétique du traduire. Lagrasse, Verdier.

- (1994). "La poésie comme contre-savoir ». Kelly Basilio et Manuel Gusmao (eds.), Poesia \& Ciência. Lisbonne, Cosmos, pp. 29-42.

- (1973). Pour la poétique II. Épistémologie de l'écriture. Poétique de la traduction. Paris, Gallimard.

OSEKI-DÉPRÉ, Inês (1999). Théories et pratiques de la traduction littéraire. Paris, Armand Colin.

PRIGOGINE, Ilya (1994). Les lois du chaos. Paris, Flammarion.

RILKE, Rainer Maria (1962 [1905]). " Das Stunden-Buch». Gesammelte Gedichte. Francfort, Insel Verlag.

- (1972 [1929]). Euvres 2. Poésie. Tr. J. Legrand, Paris, Seuil.

TABAKOWSKA, Elzbieta (1993). Cognitive Linguistics and Poetics of Translation. Tübingen, Gunter Narr Verlag.

RÉSUMÉ : La poésie pense : une modalité assomptive de la connaissance - Dans un monde utilitariste dominé par la technologie, la poésie ne correspond pas forcément aux idées qu'on a sur elle. Exploration du monde et de la vie, elle est comme la science au cœur de la connaissance, repoussant elle aussi les limites de l'inconnu et de l'impensé. Elle est encore la conscience critique du rapport nécessairement subjectif à la connaissance. C'est par son caractère " épiphanique " que la poésie se distingue du discours scientifique. Au savoir-vrai argumenté du discours de la science, elle oppose la modalité assomptive, immédiate, de la saisie du monde.

ABSTRACT : Thinking Poetry : an Assumptive Approach to Knowledge - In our technology-dominated utilitarian world, poetry does not necessarily correspond to our definitions and ideas of it. An exploration of the world and of life, it is like the science at the heart of knowledge, pushing back the boundaries of the unknown and the unthought. The critical conscience in our inescapably subjective relationship to knowledge, its "epiphanic" character is that which sets it apart from scientific discourse. Poetry opposes science's fact-based discourse on knowledge with the world's necessarily assumptive and judgmental approach to it.

Annie Brisset : École de traduction et d'interprétation, Université d'Ottawa, C.P. 450, Succ. A, Ottawa (Ontario) K1N 6N5

Courriel : abrisset@uottawa.ca 